\title{
Dynamic Combined-Mode Traffic Network Model considering Transfer Behaviors
}

\author{
ShuGuang Li $\mathbb{D I}^{1}$ and QingHua Zhou ${ }^{2}$ \\ ${ }^{1}$ School of Electronic and Control Engineering, Department of Traffic Information and Control Engineering, Chang'an University, \\ Xi'an 710064, China \\ ${ }^{2}$ School of Highway and Railway Engineering, Shaan'xi College of Communications Technology, Xi'an 710018, Shaan'xi, China \\ Correspondence should be addressed to ShuGuang Li; lxlsg@vip.sina.com
}

Received 22 February 2020; Revised 6 August 2020; Accepted 26 August 2020; Published 21 September 2020

Academic Editor: Eneko Osaba

Copyright (c) 2020 ShuGuang Li and QingHua Zhou. This is an open access article distributed under the Creative Commons Attribution License, which permits unrestricted use, distribution, and reproduction in any medium, provided the original work is properly cited.

\begin{abstract}
We propose a dynamic combined-mode traffic network model considering transfer behaviors. We assume that travelers can be classified into two classes: one class is pure-mode travelers who complete a trip by single transportation mode, and another is combined-mode travelers who cover a journey by car, bus, and so forth. The multimode point queue model is used to model the interaction of cars and buses on the network. We present an integrated variational inequality formulation to capture the complex traveler choice behaviors such as departure time choices, transfer point, and route choices. Finally, a numerical example is given to illustrate the effectiveness of the proposed heuristic algorithm and model.
\end{abstract}

\section{Introduction}

Over the past decades, many researchers have been devoted to static multimodal network models and algorithms. Recently, Bliemer [1] and Bliemer and Bovy [2] were among the first to extend a single-mode macroscopic dynamic traffic assignment model to multimode dynamic traffic model with interacting user-classes and propose various algorithms to solve the assignment problem. However, the previous models have assumed that a trip from origin to destination is completed by taking only one single mode (bus, car, etc.). Fernandez [3] denoted a trip that occurs on one single mode as a "pure-mode" trip. In recent years, with the continuous increase of the city area and the rapid growth of the traffic demand, for example, Beijing and Shanghai of China, the traveler can use multiple transportation modes to take a trip, such as a private car, bus, metro, or bike.

Meanwhile, the traffic authorities encourage people to adapt combined modes for alleviating traffic congestion by developing faster and more comfortable bus modes, in conjunction with the use of convenient transfer facilities for parking and pricing. Fernandez (1994) denoted a trip that occurs on multiple styles as a "combined-mode" trip. Thus, it is necessary to include more realistic mode choices and travel choices in the model.

García and Marín [4] proposed a bilevel program model to determine the capacities and fares of parking facilities for park'n ride trips. The lower-level program models the static multimodal traffic equilibrium problem with combined mode. The upper-level program then minimizes the total travel cost by using the capacities and fares of parking lots as control variables. Huang and $\mathrm{Li}$ [5] transformed a multimodal network to one called state-augmented multimodal system by using a state-augmented technique. In the Sam network, the numbers and kinds of transfer and nonlinear fare structures for buses can be captured. However, the application of the approach is limited due to the difficulty of the construction of the Sam network. Recently, Huang and Li [5] presented a variational inequality formulation for modeling a two-mode elastic demand transportation system with park'n ride trips.

The multiple traveler behaviors (mode, route, transfer point choice, etc.) can be integrated into a general variational inequality formulation. The heuristic algorithm based on the 
block Gauss-Seidel decomposition approach was proposed to solve the related problem. Kim et al. [6] used discrete choice modeling to develop equivalent mathematical programming formulations for the combined modal split and traffic assignment problem that explicitly considers mode and route similarities under congested networks. Ryu and Chen [7] provided an alternate weibit-based model for the combined modal split and traffic assignment problem that explicitly finds both similarities and heterogeneous perception variances under congestion. Liu et al. [8] presented a combined modal split and traffic assignment model to evaluate the impacts of park-and-ride behavior on the network flows. The models, as mentioned earlier for network equilibrium problems with combined mode, are static. It is well known that treating the problem from a static perspective might miss essential dynamic aspects of the issues. To overcome the limitation, the conceptual dynamic traffic network model with the combined mode is proposed in this paper. The ultimate aim of the proposed model is to evaluate the effects of transport policies such as improving the transfer facility and adjusting bus fare and capacity and so on.

In this paper, for the simplicity of the exposition, we study a classical combined-mode problem. We classify travelers into two classes: one is pure-mode travelers who complete a trip by the private car, and another is combinedmode travelers who take the first part of a trip by the private car from origin $r$ to parking lot $t$, walk to riding lot $t^{\prime}$, and then complete the remainder of the trip by the bus from riding lot $t$ ' to destination $s$. There exist two vehicle types in the transportation system: cars and buses. The single-mode deterministic point queue model can be extended to a multimode point queue model for modeling time and spatial interactions between cars and buses on a network.

In this paper, we use the nested-logit-type functions to capture the complex traveler choice behaviors for two classes (departure time, transfer point, route choices, etc.). A dynamic traffic network model with combined mode under hierarchical structures can be formulated in an integrated way as a variational inequality problem. The reminder of the paper is as follows. In Section 2, we propose a conceptual dynamic traffic network model with combined mode. Section 3 discusses the complex travel behaviors and offers a variational inequality formulation in an integrated way. Further, in Section 4, the heuristic algorithm is described. Finally, a numerical example is given to demonstrate the effectiveness of the proposed model and algorithm.

\section{Model Formulation}

We consider a road network $G(N, A)$ composed of a finite set of nodes, $N$, and a finite set of directed links, $A$. Let $R$ be the set of origin nodes and let $r$ represent an origin $r \in R$. Let $S$ be a set of destination nodes and let $s$ represent a destination $s \in S$. Let $a$ be a link, and let $p$ be a route, which is simply an acyclic ordered set of links, $\left\{a_{1}, a_{2}, \ldots, a_{\mathrm{n}}\right\}$, which connects an origin $r$ and a destination $s . P^{r s}$ represents the set of all feasible routes (including combined routes for combinedmode travelers) between an OD pair $(r, s)$. Denote $p b$ as a bus line composed of a finite set of the road links. $P_{b}, p b \in P_{b}$, represents the set of all bus lines. Here, two modes of transportation are explicitly considered: bus and car. We consider two kinds of link: one is the general road link used by car and bus, and another is the transfer link $\left(t, t^{\prime}\right)$ used by combined-mode travelers. Denote $\left(t, t^{\prime}\right)$ as a transfer link; here $t$ is a parking lot (transfer point) in the road network, and $t$ ' is a $t$-nearby riding lot (bus stop) in the road network. Furthermore, denote $P_{1}^{r s}$ as the set of routes used by puremode travelers between OD Pair $r s$, denote $P_{2}^{r s}$ as the set of combined routes used by combined-mode travelers between OD Pair $r s$, and $P^{r s}=P_{1}^{r s} \cup P_{2}^{r s}, \forall r s \in \mathrm{RS}$. Denote $P_{2 t}^{r s}$ as the set of combined routes via transfer point $t$ used by combined-mode travelers between OD Pair $r s$; $P_{2 t}^{r s} \in P_{2}^{r s}, \forall r s \in \mathrm{RS}$. A combined route that used $p \mathrm{t}, p t \in P_{2 t}^{r s}$, by a combined-mode traveler between OD pair $r s$ consists of three parts: the road part by car from origin $r$ to parking lot $t$ (let $p_{t}^{c}$ be the link set of the parts), the transfer part for walking from parking point $t$ to riding lot $t^{\prime}$, and the road part by bus from riding lot $t^{\prime}$ to destination $s$ (let $p_{t}^{b}$ be the link set of the parts). Then we have $p_{t}=\left\{p_{t}^{c},\left(t, t^{\prime}\right), p_{t}^{b}\right) . T_{r s}$ represents the set of all possible transfer points to be chosen between OD pair rs. Both "path" and "route" are used without difference in this paper.

The studies' horizon is discretized into $m$ intervals of length $\delta$ such that $T=m \cdot \delta$. In other words, we have intervals, and index $k$ represents the interval $[(k-1) \cdot \delta, k \cdot \delta)$. Here, it is assumed that the study horizon is long enough to ensure that all travelers can exit from the network after the time T. On the other hand, it is also assumed that the value of $\delta$ is small enough so that the discrete-time model can approximate its continuous-time counterpart. Further, it is assumed that the flow rate, specified either by link or by route, is constant during a given interval.

2.1. Dynamic Network Model. Li and Su, [9] and Li and Ju [10] proposed a multimode stochastic dynamic simultaneous route/departure time equilibrium model and proved that the multimode deterministic point model within each mode-user meets the FIFO discipline, and the speeds of different modes approach consistency during congestion. Each link consists of two distinct segments. The first segment is the running segment of the link on which car (bus) can run according to the free-flow velocity of cars (buses). In other words, cars (buses) experience the constant running time $t_{a}^{c}\left(t_{a}^{b}\right)$ to the exit queue segment of link. The second segment is the exit queue segment (car and bus are assumed to be a point without length). The queue delay experienced by cars (buses) is caused by the limited link exit capacity (in Pcu) or the maximum link exit flow rate (in $P c u / h$ ).

2.1.1. Link Dynamic Model. The traffic flow of cars (buses), $u_{a}^{c}\left(k_{1}\right)\left(u_{a}^{b}\left(k_{1}\right)\right)$, entering link $a$ during interval $k_{1}$, experiences the constant running time $t_{a}^{c}\left(t_{a}^{b}\right)$ to arrive at the exit queue of the link, and the arrival flow rate of cars (buses) to the exit queue segment during interval $k$ on link $a$ is $u_{a}^{c}\left(k-t_{a}^{c}\right)\left(u_{a}^{b}\left(k-t_{a}^{b}\right)\right)$. The departure flow rate of cars (buses) from the exit queue segment during interval $k$ is 
$v_{a}^{c}(k)\left(v_{a}^{b}(k)\right)$. Thus, the link dynamic model on link $a$ can be formulated as follows:

$$
\begin{array}{ll}
\frac{q_{a}^{c}(k)-q_{a}^{c}(k-1)}{\delta}=u_{a}^{c}\left(k-t_{a}^{c}\right)-v_{a}^{c}(k), & \forall a, k, \\
\frac{q_{a}^{b}(k)-q_{a}^{b}(k-1)}{\delta}=u_{a}^{b}\left(k-t_{a}^{b}\right)-v_{a}^{b}(k), & \forall a, k .
\end{array}
$$

where $q_{a}^{c}(k)\left(q_{a}^{b}(k)\right)$ represents the queue length of cars (bus) at the bottleneck of link an at interval $k$. Equations (1) and (2) show that the queue length marginal change of cars (bus) is equal to the difference between the arrival flow rate of cars (bus) to exit queue segment during interval $k-t_{a}^{c}(k-$ $\left.t_{a}^{b}\right)$ and the departure flow rate of cars (bus) from exit queue segment during interval $k$ on link $a[9,10]$.

2.1.2. Link Exit Model. The temporal and spatial interactions of cars and buses mainly appear in the exit queue segment of the link, if the sum of queue lengths of cars and bus, $q_{a}(k)$ (here, let $q_{a}(k)=\mathrm{pcu} \cdot q_{a}^{c}(k)+\mathrm{pcu}_{b} \cdot q_{a}^{b}(k)$, in passenger car units) is equal to zero. In other words, cars and bus will pass the exit queue without delay. According to nonnegativity of the queue lengths of cars and bus, we can obtain $q_{a}^{c}(k)=0, q_{a}^{b}(k)=0$. Substituting it into equations (1) and (2), we can obtain

$$
\begin{array}{ll}
v_{a}^{c}(k)=u_{a}^{c}\left(k-t_{a}^{c}\right)+\frac{q_{a}^{c}(k-1)}{\delta}, & \forall a, k, \\
v_{a}^{b}(k)=u_{a}^{b}\left(k-t_{a}^{b}\right)+\frac{q_{a}^{b}(k-1)}{\delta}, & \forall a, k .
\end{array}
$$

If $q_{a}(k)>0$ or the sum of arrival flow rates of cars and buses to the exit queue segment $v_{a}(k)=\mathrm{pcu} \cdot u_{a}^{c}\left(k-t_{a}^{c}\right)+$ $\mathrm{pcu}_{b} \cdot u_{a}^{b}\left(k-t_{a}^{b}\right)+\left(\mathrm{pcu} \cdot q_{a}^{c}(k-1)+\mathrm{pcu}_{b} \cdot q_{a}^{b}(k-1)\right) / \delta$ is more than $s_{\mathrm{a}}$, then the arrival flow rate to the exit queue segment must not wait, and the flow rate exiting from the link exit queue segment is $s_{\mathrm{a}}$. According to the assumption in (2), the link flow rates of cars and buses exiting from the exit queue segment can be calculated as follows:

$$
\begin{array}{ll}
v_{a}^{c}(k)=\frac{u_{a}^{c}\left(k-t_{a}^{c}\right)+q_{a}^{c}(k-1) / \delta}{v_{a}(k)} \cdot s_{a}, & \forall a, k, \\
v_{a}^{b}(k)=\frac{u_{a}^{b}\left(k-t_{a}^{b}\right)+q_{a}^{b}(k-1) / \delta}{v_{a}(k)} \cdot s_{a}, & \forall a, k .
\end{array}
$$

Furthermore, equations (1) and (2) can be rewritten, respectively, as follows:

$$
\begin{aligned}
& q_{a}^{c}(k)= \begin{cases}u_{a}^{c}\left(k-t_{a}^{c}\right) \cdot \delta+q_{a}^{c}(k-1)-\frac{u_{a}^{c}(k) \cdot \delta+q_{a}^{c}(k-1)}{v_{a}(k)} \cdot s_{a}, & \text { if } v_{a}(k) \geq s_{a}, \\
0, & \text { otherwise, } \\
q_{a}^{b}(k)=\left\{\begin{array}{ll}
u_{a}^{b}\left(k-t_{a}^{b}\right) \cdot \delta+q_{a}^{b}(k-1)-\frac{u_{a}^{b}(k) \cdot \delta+q_{a}^{b}(k-1)}{v_{a}(k)} \cdot s_{a}, & \text { if } v_{a}(k) \geq s_{a}, \\
0, & \text { otherwise. }
\end{array} \quad \forall a, k,\right.\end{cases}
\end{aligned}
$$

2.1.3. Link Travel Time. The total link travel times for cars and buses entering link $a$ during interval $k$ are the sum of the travel times of the running segment and the queue delay, as given below.

$$
t_{a}^{b}(k)=t_{a}^{b}+d_{a}^{b}(k), t_{a}^{c}(k)=t_{a}^{c}+d_{a}^{c}(k) \quad \forall a, k,
$$

where

$$
d_{a}^{c}(k)=\frac{q_{a}\left(k+t_{a}^{c}\right)}{s_{a}}, \quad \forall a, k,
$$

$$
d_{a}^{b}(k)=\frac{q_{a}\left(k+t_{a}^{b}\right)}{s_{a}},
$$

where $d_{a}^{c}(k)$ and $d_{a}^{b}(k)$ express the queue delays of cars and buses entering link $a$ during interval $k$ which are dependent on the link total queue length, respectively. The cars and buses entering link $a$ during interval $k$ will spend the travel time $t_{a}^{c}, t_{a}^{b}$ to arrive at the exit queue. 


$$
\begin{gathered}
u f_{a p}^{r s}(k)= \begin{cases}f_{p}^{r s}(k), & a \text { is first link on route } p, \quad \forall p \in P_{1}^{r s}, \forall k \in K, \forall a \in p, \forall r s \in \mathrm{RS}, \\
v f_{b p}^{r s}(k), & b \text { is link preceding link a on route } p,\end{cases} \\
u f_{\text {aqt }}^{r s}(k)= \begin{cases}f_{q t}^{r s}(k), & a \text { is first link on route } q t, \\
v f_{b q t}^{r s}(k), & b \text { is link preceding link a on route } q t,\end{cases}
\end{gathered}
$$

where equations (12) and (13) depict the flow conservation constraints of pure-mode and combined-mode travelers at the origin and the other nodes, respectively. $u f_{a p}^{r s}(k)$ and $v f_{a p}^{r s}(k)$ are the inflow and departure flow rate of pure-mode travelers entering link $a$ of route $p$ between OD pair $r s$ during interval $k$, respectively; $u f_{a q t}^{r s}(k)$ and $v f_{\text {aqt }}^{r s}(k)$ are the inflow and departure flow rate of combined-mode travelers entering link $a$ of route $q$ via transfer point $t$ between OD pair $r s$ during interval $k$, respectively; $f_{p}^{r s}(k)$ represents the inflow rate of puremode travelers entering route $p$ between OD pair $r s$ during interval $k . f_{q t}^{r s}(k)$ represents the inflow rate of combinedmode travelers entering route $q$ via transfer point $t$ between OD pair $r$ s during interval $k$.

The flow conservation of buses can be expressed as follows:

$$
u_{a p b}(k)= \begin{cases}F_{p b}, & a \text { is first link on bus line } p b, \quad \forall a \in p b, \forall p b \in P_{b}, \forall k \in K, \\ v_{d p b}(k), & d \text { is link preceding link a on bus line } p b\end{cases}
$$

where $u_{a p b}(k)$ is the flow rate of buses entering link $a$ of bus line $p b$, which is the general road link during interval $k$. $v_{d p b}(k)$ is the flow rate of buses exiting link $d$ of bus line $p b$ during interval $k$. Link $d$ is the predecessor of link $a$ on bus line $p b$. When link $a$ is first link on bus line $p b$, the inflow rate on link $a$ of bus line $p b$ is equal to the bus dispatching frequency of bus line $p b, F_{p b}$.

2.1.5. The Route-Specific Link Flow. The traveler flow rates on the link can be given as follows:

$$
\begin{aligned}
& u f_{a}^{t}(k)=\sum_{r s \in R S} \sum_{p t} u f_{a p t}^{r s}(k), \quad \forall a \in\left(t, t^{\prime}\right), k \in K, \\
& u f_{a}^{b}(k)=\sum_{r s \in R S} \sum_{t} \sum_{p t} u f_{a p t}^{r s}(k), \quad \forall a \in p_{t}^{b}, k \in K, \\
& u f_{a}^{c}(k)=\sum_{r s} \sum_{p} u f_{a p}^{r s}(k)+\sum_{r s} \sum_{t} \sum_{p t} u f_{a p t}^{r s}(k) \cdot \psi_{a, p t}, \quad \forall a \in L, k \in K, u f_{a}^{t}(k),
\end{aligned}
$$

where $u f_{a}^{t}(k)$ represents the flow rate of combined-mode travelers entering transfer link $\left(t, t^{\prime}\right)$ during interval $k$. $u f_{a}^{b}(k)$ represents the flow rate of combined-mode travelers on bus entering link $a$ during interval $k . u f_{a}^{c}(k)$ describes the flow rate of travelers (including combinedmode and pure-mode travelers) on the car entering link $a$ during interval $k . \psi_{a, p t}=1$ if link $a$ lies on the link before transfer link $\left(t, t^{\prime}\right)$ of route $p t, a \in p_{t}^{c}$, and $\psi_{a, p t}=0$ otherwise, $a \in p_{t}^{b}$. The first term on the right side of equation (17) expresses the flow rate of pure-mode travelers on link $a$ during interval $k$. The second term on the right side of equation (17) expresses the flow rate of combined-mode travelers by the car at the first part of a trip on link $a$ during interval $k$. 
The link inflow rates of cars and buses can be expressed as follows:

$$
\begin{gathered}
u_{a}^{c}(k)=u f_{a}^{c}(k) / \Phi_{r s}, \quad \forall a \in L, k \in K, \\
u_{a}^{b}(k)=\sum_{p b \in P_{b}} u_{a p b}(k), \forall a \in L, k \in K
\end{gathered}
$$

where $\omega_{r s}$ is the fixed average car occupancy rate between OD pair $r s$.

The following relationship must hold to satisfy the FIFO within each mode (car and bus):

$$
\begin{aligned}
& v_{a p b}(k)=\frac{u_{a p b}\left(k_{1}\right)}{u_{a}^{b}\left(k_{1}\right)} v_{a}^{b}(k), k_{1}+t_{a}^{b}\left(k_{1}\right)=k, \quad \forall a \in L, \forall k \in K, \forall p b \in P_{b}, \\
& v_{a p c}^{r s}(k)=\frac{u_{a p c}^{r s}\left(k_{1}\right)}{u_{a}^{c}\left(k_{1}\right)} v_{a}^{c}(k), k_{1}+t_{a}^{c}\left(k_{1}\right)=k, \quad \forall a \in L, \forall k \in K, \forall p \in P^{r s}, \forall r s \in R S,
\end{aligned}
$$

where $u_{a p c}^{r s}(k)$ and $v_{a p c}^{r s}(k)$ are the inflow and departure flow rates of cars on link $a$ of route $p$ between OD pair $r s$ during interval $k$, respectively. $u_{a p c}^{r s}(k)=u f_{a p}^{r s}(k) / \omega_{r s}$ or $u f_{a p t}^{r s}(k) / \Phi_{r s}$. Equations (20) and (21) represent the ways of calculation of the route-specific link flow rate, respectively. A similar way was used to determine the route-specific or destination-specific link flow rate $[11,12]$. Here, we assume that cars and buses belonging to different routes are randomly mixed in the link provided that they arrive at the link at the same interval.

Substituting $u_{a p c}^{r s}(k)=u f_{a p}^{r s}(k) / \Phi_{r s}$ or $u f_{a p t}^{r s}(k) / \Phi_{r s}$ into equation (21), the route-specific flow rates for pure-mode and combined-mode travelers by private car on link $a$ can be calculated as follows:

$$
\begin{aligned}
& v f_{a p}^{r s}(k)=\frac{u f_{a p}^{r s}\left(k_{1}\right)}{u_{a}^{c}\left(k_{1}\right)} v_{a}^{c}(k), k_{1}+t_{a}^{c}\left(k_{1}\right)=k, \quad \forall a \in L, \forall k \in K, \forall p \in P_{1}^{r s}, \forall r s \in R S, \\
& v f_{a p t}^{r s}(k)=\frac{u f_{a p t}^{r s}\left(k_{1}\right)}{u_{a}^{c}\left(k_{1}\right)} v_{a}^{c}(k), k_{1}+t_{a}^{c}\left(k_{1}\right)=k, \quad \forall a \in p_{t}^{c}, k \in K, p t \in P_{2 t}^{r s}, t \in T^{r s}, r s \in R S,
\end{aligned}
$$

where equations (22) and (23) represent the ways of calculation of the route-specific link flow rates associated with pure-mode travelers and combined-mode travelers using cars on link $a$. Furthermore, we assume that the pure-mode and combined-mode travelers on different routes randomly distribute themselves among cars entering link $a$ at the same interval.

Similar to equations (22) and (23), the route-specific link flow rate of combined-mode travelers on buses can be expressed as follows:

$$
v f_{a p t}^{r s}(k)=\frac{u f_{a p t}^{r s}\left(k_{1}\right)}{u_{a}^{b}\left(k_{1}\right)} v_{a}^{b}(k), k_{1}+t_{a}^{b}\left(k_{1}\right)=k, \quad \forall a \in p t, k \in K, p t \in P_{2 t}^{r s}, t \in T^{r s}, r s \in \mathrm{RS} .
$$

Here, we assume that the combined-mode travelers on different combined routes randomly distribute themselves among buses entering link $a$ at the same interval.

2.1.6. Transfer Link. Huang and Li [5] proposed several different ways to model the transfer behavior of travelers on the transfer link. In this section, the travel times experienced by combined-mode travelers on the transfer link can be calculated based on the point queue model.
Firstly, we assume that the exit capacity of the transfer link, $s_{a}$, is the time-invariant constant. Combined-mode travelers entering transfer link $\left(t, t^{\prime}\right)$ experience the constant travel time $t_{a}^{t}$ (including parking time, walking time from parking lot to riding lot $t$, and average waiting time for the bus at bus stop $t^{\prime}$ ) to arrive at bus stop $t^{\prime}$. Due to the limited exit capacity of transfer link $\left(t, t^{\prime}\right)$, the combined-mode travelers arriving at bus stop $t$ ' must not wait and form a queue. 
The departure flow rate of combined-mode travelers on transfer link $\left(t, t^{\prime}\right)$ is given as follows:

$$
v f_{a}^{t}(k)=\left\{\begin{array}{ll}
u f_{a}^{t}\left(k-t_{a}^{t}\right)+q_{a}^{t}(k-1) / \delta, & \text { if } u f_{a}^{t}\left(k-t_{a}^{t}\right)<s_{a}, \\
s_{a}, & \text { otherwise, }
\end{array} \quad \forall a \in\left(t, t^{\prime}\right), k\right.
$$

The link queue model on the transfer link can be expressed as follows:

$$
q_{a}^{t}(k)=\left\{\begin{array}{ll}
\left(u f_{a}^{t}\left(k-t_{a}^{t}\right)-s_{a}\right) \cdot \delta+q_{a}^{t}(k-1), & \text { if } u f_{a}^{t}\left(k-t_{a}^{t}\right) \geq s_{a}, \\
0, & \text { otherwise, }
\end{array} \quad \forall a \in\left(t, t^{\prime}\right), k .\right.
$$

where $q_{a}^{t}(k)$ is the number of combined-mode travelers waiting at the bus stop (riding lot) $t^{\prime}$ on transfer link $\left(t, t^{\prime}\right)$ at interval $k$.

The total travel time over link $a$ for a combined-mode traveler entering transfer link during interval $k$ is given as follows:

$$
t_{a}^{t}(k)=t_{a}^{t}+\frac{q_{a}^{t}\left(k+t_{a}^{t}\right)}{s_{a}}, \quad \forall a \in\left(t, t^{\prime}\right), k
$$

$$
v f_{a p t}^{r s}(k)=\frac{u f_{a p t}^{r s}\left(k_{1}\right)}{u_{a}^{t}\left(k_{1}\right)} v_{a}^{t}(k), k_{1}+t_{a}^{t}\left(k_{1}\right)=k
$$

where we assume that the combined-mode travelers belonging to different routes are randomly mixed in the queue of bus stop provided that they arrive at the transfer link at the same interval. $u_{a p t}^{r s}\left(k_{1}\right)$ and $v_{a p t}^{r s}\left(k_{1}\right)\left(a \in\left(t, t_{\prime}\right)\right)$ are the inflow rate and departure flow rate of combined-mode travelers entering transfer link $\left(t, t^{\prime}\right)$ of route $p t$ between OD pair $r s$ during interval $k$, respectively.

In the above studies, we use multimode dynamic traffic flow model to model the interaction of vehicles such as cars and buses. Further, the conversion between the traveler flow and the vehicle flow can be realized at the route-specific link flow and transfer link, and so forth. So the problem with the parallel paths for buses can be solved.

2.1.7. Link Travel Cost and Route Travel Cost. The travel costs for different travelers can be given on link $a$, which is either a road link or transfer link, as follows. Also, we define the unit of travel cost in money terms.

$$
\begin{aligned}
& c_{a}^{t}(k)=\gamma_{t} \cdot t_{a}^{t}(k)+\rho_{t}, \quad \forall a \in\left(t, t^{\prime}\right), k, \\
& c_{a}^{c}(k)=\alpha_{1} \cdot t_{a}^{c}(k), \quad \forall a \in L, k \in K,
\end{aligned}
$$

The second term on the right side of the above equation expresses the overload delay time at the bus stop due to the limited capacity of the transfer link.

The following equation can give the route-specific link flow rate of combined-mode travelers to satisfy the FIFO on transfer link $\left(t, t^{\prime}\right)$ similar to equations (22)-(24):

$\forall a \in\left(t, t^{\prime}\right), \forall k \in K, \forall p t \in P_{2}^{r s}, \forall r s \in \mathrm{RS}$

$$
c_{a}^{b}(k)=\alpha_{2} \cdot t_{a}^{b}(k)+\lambda_{2}\left(\frac{u f_{a}^{b}(k)}{u_{a}^{b}(k) \cdot c_{b}}\right)^{2} \quad \forall a \in L, k
$$

where equation (29) represents the travel cost incurred by combined-mode travelers entering transfer link $\left(t, t^{\prime}\right)$ during interval $k$. Equation (30) represents the travel cost incurred by travelers (including pure-mode and combined-mode travelers) by a private car entering link $a$ during interval $k$. Equation (31) represents the travel cost incurred by combined-mode travelers by bus entering link $a$ during interval $k$, where $\gamma_{t}, \alpha_{1}$, and $\alpha_{2}$ are the coefficients that transform the travel time into the travel cost, $\lambda_{2}$ is a conversion coefficient too, and the parameter $\rho_{t}$ is the fare (RMB) associated with transfer link $\left(t, t^{\prime}\right)$ such as parking fare. The second term $\left(u f_{a}^{b}(k) / u_{a}^{b}(k) \cdot c_{b}\right)^{2}$ on the right side of equation (31) expresses the discomfort that is experienced by a combined-mode traveler by bus on a link $a$.

Now we use the nested function to establish the route travel times and route travel costs for pure-mode and combined-mode travelers. Note that the calculation of route travel time and travel cost for pure-mode travelers is different from that for combined-mode travelers. The travel times required to traverse route $p=\left\{a_{1}, a_{2}, \ldots, a_{\mathrm{n}}\right\}$ 
for pure-mode travelers entering the network during interval $k$ can be given as follows:

$$
\begin{aligned}
t_{p}^{r s}(k)= & t_{a_{1}}^{c}(k)+t_{a_{2}}^{c}\left(k+t_{a_{1}}^{c}(k)\right)+\cdots \\
& +t_{a_{n}}^{c}\left(k+t_{a_{1}}^{c}+t_{a_{2}}^{c}+\cdots+t_{a_{n-11}}^{c}\right), \quad \forall r s \in \mathrm{RS}, p \in P_{1}^{r s},
\end{aligned}
$$

where $t_{a_{1}}^{c}=t_{a_{1}}^{c}(k), t_{a_{2}}^{c}=t_{a_{2}}^{c}\left(k+t_{a_{1}}^{c}(k)\right)$. . for short.

The route travel cost experienced by a pure-mode traveler entering route $p$ between OD pair $r s$ during interval $k$ is given as follows:

$$
\begin{aligned}
c_{p}^{r s}(k)= & c_{a_{1}}^{c}(k)+c_{a_{2}}^{c}\left(k+t_{a_{1}}^{c}(k)\right)+\cdots \\
& +c_{a_{n}}^{c}\left(k+t_{a_{1}}^{c}+t_{a_{2}}^{c}+\cdots+t_{a_{n-1}}^{c}\right), \quad \forall r s \in \mathrm{RS}, p \in P_{1}^{r s} .
\end{aligned}
$$

The route travel time and route travel cost for a combined-mode traveler can be classified into three sections associated with the three parts of the combined route (from origin $r$ to parking lot $t$ by car, from $t$ to riding point $t$ ' by walk, and from $t^{\prime}$ to destination $s$ by bus). Thus, the travel time required to traverse route $p t=\left\{a_{1}, a_{2}, \ldots, a_{\mathrm{t}},\left(t, t^{\prime}\right), a_{\mathrm{t}^{\prime}}, \ldots\right.$, $\left.a_{\mathrm{n}}\right\}$ for combined-mode travelers entering the network during interval $k$ can be given as follows:

$$
\begin{aligned}
t_{p t}^{r s}(k)= & t_{a_{1}}^{c}(k)+t_{a_{2}}^{c}\left(k+t_{a_{1}}^{c}(k)\right)+\cdots \\
& +t_{a_{t}}^{c}\left(k+t_{a_{1}}^{c}+t_{a_{2}}^{c}+\cdots+t_{a_{t-1}}^{c}\right) \\
& +t_{a_{t}}^{t}\left(k+t_{a_{1}}^{c}+t_{a_{2}}^{c}+\cdots+t_{a_{t}}^{c}\right) \\
& +t_{a_{t}}^{b}\left(k+t_{a_{1}}^{c}+t_{a_{2}}^{c}+\cdots+t_{a_{t}}^{c}+t_{a_{t}}^{t}\right)+\cdots \\
& +t_{a_{t}}^{b}\left(k+t_{a_{1}}^{c}+t_{a_{2}}^{c}+\cdots+t_{a_{t}}^{c}+t_{a_{t}}^{t}+t_{a_{t}}^{b}+\cdots+t_{a_{n-1}}^{b}\right), \quad \forall p \in P_{2}^{r s} .
\end{aligned}
$$

The route travel cost experienced by a combined-mode traveler entering route $p t$ via transfer point $t$ between OD pair $r s$ during interval $k$ can be expressed as follows:

$$
\begin{aligned}
c_{p t}^{r s}(k)= & c_{a_{1}}^{c}(k)+c_{a_{2}}^{c}\left(k+t_{a_{1}}^{c}(k)\right)+\cdots \\
& +c_{a_{t}}^{c}\left(k+t_{a_{1}}^{c}+t_{a_{2}}^{c}+\cdots+t_{a_{t-1}}^{c}\right) \\
& +c_{a_{t}}^{t}\left(k+t_{a_{1}}^{c}+t_{a_{2}}^{c}+\cdots+t_{a_{t}}^{c}\right) \\
& +c_{a_{t}}^{b}\left(k+t_{a_{1}}^{c}+t_{a_{2}}^{c}+\cdots+t_{a_{t}}^{c}+t_{a_{t}}^{t}\right)+\cdots \\
& +c_{a_{t}}^{b}\left(k+t_{a_{1}}^{c}+t_{a_{2}}^{c}+\cdots+t_{a_{t}}^{c}+t_{a_{t}}^{t}+t_{a_{t}}^{b}+\cdots+t_{a_{n-1}}^{b}\right), \quad \forall p \in P_{2}^{r s},
\end{aligned}
$$

Considering the schedule delay costs of arriving at destinations as well as bus fare, we give the generalized route travel cost functions for pure-mode and combined-mode travelers as follows:

$$
\begin{aligned}
& C_{p}^{r s}\left(k, f, f_{t}\right)=c_{p}^{r s}(k)+ \begin{cases}\beta \cdot\left[k_{r s}^{*}-\Delta_{r s}-k-t_{p}^{r s}(k)\right], & \text { if } k+t_{p}^{r s}(k)<k_{r s}^{*}-\Delta_{r s}, \\
\gamma \cdot\left[k+t_{p}^{r s}(k)-k_{r s}^{*}-\Delta_{r s}\right], & \text { if } k+t_{p}^{r s}(k)>k_{r s}^{*}+\Delta_{r s}, \\
0, & \text { otherwise, }\end{cases} \\
& C_{p t}^{r s}\left(k, f, f_{t}\right)=c_{p t}^{r s}(k)+\lambda_{b} \cdot \rho_{t, s, b}+ \begin{cases}\beta \cdot\left[k_{r s}^{*}-\Delta_{r s}-k-t_{p t}^{r s}(k)\right], & \text { if } k+t_{p t}^{r s}(k)<k_{r s}^{*}-\Delta_{r s}, \\
\gamma \cdot\left[k+t_{p t}^{r s}(k)-k_{r s}^{*}-\Delta_{r s}\right], & \text { if } k+t_{p t}^{r s}(k)>k_{r s}^{*}+\Delta_{r s}, \\
0, & \text { otherwise, }\end{cases}
\end{aligned}
$$

where $\beta$ is the unit cost of schedule delay time-early, $\gamma$ is the unit cost of schedule delay time-late, and $\left[k_{r s}^{*}-\Delta, k_{r s}^{*}+\Delta\right]$ is the window of arrival times at destination $s . k_{r s}^{*}$ is middle point of the time window and may represent the official work start time for trips between OD pair $r s . \rho_{t_{1} s, b}$ in equation (37) represents the nonadditive bus fare from riding lot (bus stop) $t^{\prime}$ to destination $s$ and $\lambda_{b}$ is a conversion parameter. $f\left(f_{t}\right)$ is the vector of all route inflow rates for pure-mode (combined-mode) travelers.

2.2. Traveler Choices. Figure 1 shows the nested choice process of travelers during regular peak hour periods. Here, we assume that the travel choice process can be broken down into a sequence of mobility choices represented by a choice tree. Pure-mode travelers who complete a trip by car first decide what time to depart and then which route to follow; otherwise, combined-mode travelers who complete a trip by car and bus first determine what time to leave and then what transfer link to choose and finally which route via transfer link $\left(t, t^{\prime}\right)$ to follow.

2.2.1. The Traveler Choices for Pure-Mode Travelers. For pure-mode travelers, their route and departure time choices are random to a certain extent because they only have partial information for decision-making. Here, we assume that a pure-mode traveler first chooses what time 

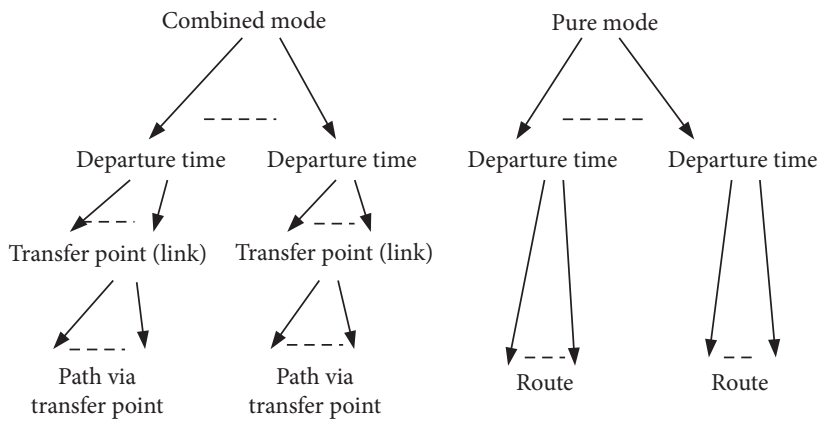

FIgURE 1: The nested choice process of travelers.

to depart and then which route to follow. A nested-logit formulation is used in modeling the sequential decisionmaking process. The behavior that a pure-mode traveler departing during interval $k$ between OD pair $r s$ selects route $p$ can be expressed as follows:

$$
f_{p}^{r s}(k)=q e_{1}^{r s}(k) \frac{\exp \left(-\theta_{R}^{r s} \cdot C_{p}^{r s}(k, \cdot)\right)}{\sum_{p \in P_{1}^{r s}} \exp \left(-\theta_{R}^{r s} \cdot C_{p}^{r s}(k, \cdot)\right)}, \quad \forall p \in P_{1}^{r s}, \forall r s \in \mathrm{RS}, \forall k \in K,
$$

where $q e_{1}^{r s}(k)$ is the number of pure-mode travelers during interval $k$ between OD pair $r s . \theta_{R}^{r s}$ is the parameter calibrated on observed data that reflect the route travel cost uncertainty on route choices of pure-mode travelers. The larger the value of $\theta_{R}^{r s}$ is, the smaller the perception error to route travel cost is. When $\theta_{R}^{r s}$ leads to infinity, a pure-mode traveler will tend to select the route with minimum generalized unit travel cost.

The expected minimum costs (EMC) for pure-mode travelers departing during interval $k$ between OD pair $r s$ can be given as follows:

$$
\begin{aligned}
\mu_{1}^{r s}(k) & =-\frac{1}{\theta_{R}^{r s}} \ln \left(\sum_{p \in P_{1}^{r s}} \exp \left(-\theta_{R}^{r s} \cdot C_{p}^{r s}(k)\right)\right) \\
& =C_{p}^{r s}(k)+\frac{1}{\theta_{R}^{r s}} \ln \left(\frac{f_{p}^{r s}(k)}{q e_{1}^{r s}(k)}\right), \quad \forall r s \in \mathrm{RS}, \forall k \in K .
\end{aligned}
$$

The departure time choice behaviors for pure-mode travelers can be described as follows:

$$
q e_{1}^{r s}(k)=q e_{1}^{r s} \frac{\exp \left(-\theta_{D}^{r s} \cdot \mu_{1}^{r s}(k)\right)}{\sum_{k \in K} \exp \left(-\theta_{D}^{r s} \cdot \mu_{1}^{r s}(k)\right)}, \quad \forall r s \in \mathrm{RS}, \forall k \in K,
$$

where $q e_{1}^{r s}$ is the number of pure-mode travelers between OD pair $r s$. The parameter $\theta_{D}^{r s}$ reflects the travel cost sensitivity on departure time choices. To ensure the validity of formulations (38)-(40), $\theta_{R}^{r s} \geq \theta_{D}^{r s}$ must hold.
The EMC for pure-mode travelers between OD pair $r s$ is given as follows:

$$
\begin{aligned}
\mu_{1}^{r s} & =-\frac{1}{\theta_{D}^{r s}} \ln \left(\sum_{p \in P_{1}^{r s}} \exp \left(-\theta_{D}^{r s} \cdot \mu_{1}^{r s}(k)\right)\right) \\
& =\mu_{1}^{r s}(k)+\frac{1}{\theta_{D}^{r s}} \ln \left(\frac{q e_{1}^{r s}(k) \cdot \delta}{q_{1}^{r s}}\right), \quad \forall r s \in R S .
\end{aligned}
$$

Substituting equation (39) and then equation (38) into equation (41), we can obtain

$$
\begin{aligned}
\mu_{1}^{r s}= & C_{p}^{r s}(k, \cdot)+\frac{1}{\theta_{R}^{r s}} \ln \left(\frac{f_{p}^{r s}(k)}{q_{1}^{r s}(k)}\right)+\frac{1}{\theta_{D}^{r s}} \ln \left(\frac{q e_{1}^{r s}(k) \cdot \delta}{q_{1}^{r s}}\right) \\
= & \frac{\theta_{R}^{r s}}{\theta_{D}^{r s}} \cdot C_{p}^{r s}(k, \cdot)+\frac{\theta_{R}^{r s}-\theta_{D}^{r s}}{\theta_{D}^{r s} \cdot \theta_{R}^{r s}} \ln \left(\sum_{p \in P_{1}^{r s}} \exp \left(-\theta_{R}^{r s} \cdot C_{p}^{r s}(k, \cdot)\right)\right) \\
& +\frac{1}{\theta_{D}^{r s}} \ln \frac{f_{p}^{r s}(k) \cdot \delta}{q e_{1}^{r s}} .
\end{aligned}
$$

The above equation is similar to the result derived by Huang and Li [5]. Here, following Huang and Li [5], for each generalized unit route travel cost of pure-mode travelers $C_{p}^{r s}(k)$, we define a so-called augmented travel cost that is equal to the right-hand side of equation (42). 


$$
\widehat{C}_{p}^{r s}(k, \cdot)=\frac{\theta_{R}^{r s}}{\theta_{T}^{r s}} \cdot C_{p}^{r s}(k, \cdot)+\frac{\theta_{R}^{r s}-\theta_{T}^{r s}}{\theta_{T}^{r s} \cdot \theta_{R}^{r s}} \ln \left(\sum_{p \in P_{1}^{r s}} \exp \left(-\theta_{R}^{r s} \cdot C_{p}^{r s}(k, \cdot)\right)\right)+\frac{1}{\theta_{T}^{r s}} \ln \frac{f_{p}^{r s}(k) \cdot \delta}{q e_{1}^{r s}} .
$$

Lam and Huang [13] indicate the equalization of augmented travel cost $\widehat{C}_{p}^{r s}(k)$ for all used routes $p$ and selected departure time $k$ implies a nested-logit stochastic dynamic route and departure time equilibrium solution as given by equations (38)-(40).
Therefore, the nested-logit stochastic dynamic route and departure time equilibrium condition for pure-mode travelers can be given as follows:

$$
\widehat{C}_{p}^{r s}(k, \cdot)\left\{\begin{array}{l}
=\hat{C}_{\text {min }}^{r s}, \text { if } f_{p}^{r s}(k)>0 \\
>\hat{C}_{\text {min }}^{r s}, \text { if } f_{p}^{r s}(k)=0
\end{array} \quad \forall p \in P_{1}^{r s}, k \in K, r s \in \mathrm{RS},\right.
$$

where $\widehat{C}_{\min }^{r s}=\min \left\{\widehat{C}_{p}^{r s}(k, \cdot), p \in P_{1}^{r s}, k \in K\right\}$. Under the equilibrium condition, the augmented travel cost experienced by all pure-mode travelers between OD pair $r s$ is equal and minimum and less than (or equal to) the expanded travel cost for pure-mode travelers on any unused routes. Pure-mode travelers cannot modify departure time and route choices to diminish the expanded travel cost.

The above SUE-SRD equilibrium condition of puremode travelers can be expressed by a finite-dimensional variational inequality formulation.

Find a vector $\widehat{f}^{*} \in \Omega$ if and only if it satisfies

$$
\sum_{r s} \sum_{p} \sum_{k} \widehat{C}_{p}^{r s}\left(k, f^{*}, f_{t}\right)\left(f_{p}^{r s}(k)-f_{p}^{r s *}(k)\right) \geq 0, \quad \forall f \in \widehat{\Omega},
$$

where $\widehat{\Omega}$ is a closed convex.

$$
\widehat{\Omega}=\left\{f \mid \sum_{\mathrm{p}} \sum_{\mathrm{k}} f_{p}^{r s *}(k)=\frac{q e_{1}^{\mathrm{rs}}}{\delta}, f_{p}^{r s}(k) \geq 0, \quad \forall r s\right\} .
$$

2.2.2. The Traveler Choices for Combined-Mode Travelers. A combined-mode traveler uses the car in the first part of the trip, walks at the transfer link, and then uses the bus to complete the trip. Here, we assume that a combined-mode traveler first decides what time to depart and then which transfer point to choose and finally which route via transfer point to follow. Similar to pure-mode travelers' travel choice analysis, the sequential decision-making process of combined-mode travelers results in a nested-logit formulation. The behavior that a combined-mode traveler departing during interval $k$ between OD pair $r s$ selects route $p$ via transfer point $t$ can be depicted as follows:

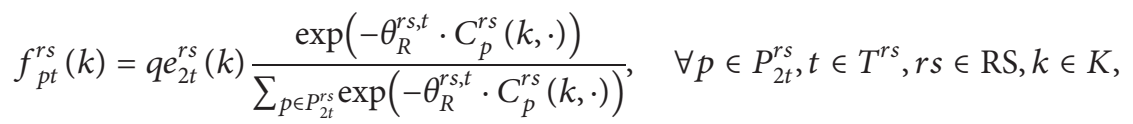

where $q e_{2 t}^{r s}(k)$ is the number of combined-mode travelers during interval $k$ between OD pair $r s$ via transfer point $t$. $\theta_{R}^{r s, t}$ is the parameter that reflects the travel cost sensitivity on route choices of combined-mode travelers.
The EMC for combined-mode travelers during interval $k$ between OD pair $r s$ via transfer point $t$ is given as follows:

$$
\mu_{2 t}^{r s}(k)=-\frac{1}{\theta_{R}^{r s, t}} \ln \left(\sum_{p \in P_{2 t}^{r s}} \exp \left(-\theta_{R}^{r s, t} \cdot C_{p}^{r s}(k, \cdot)\right)\right)=C_{p t}^{r s}(k, \cdot)+\frac{1}{\theta_{R}^{r s, t}} \ln \left(\frac{f_{p t}^{r s}(k)}{q e_{2 t}^{r s}(k)}\right) \quad \forall t \in T^{r s}, r s \in \mathrm{RS}, k \in K .
$$


The transfer point choice behaviors for combined-mode travelers can be depicted as follows:

$$
q e_{2 t}^{r s}(k)=q e_{2}^{r s}(k) \frac{\exp \left(-\theta_{T}^{r s, t} \cdot\left(\mu_{2 t}^{r s}(k)-\chi_{t}^{r s}\right)\right)}{\sum_{t \in T^{r s}} \exp \left(-\theta_{T}^{r s, t} \cdot\left(\mu_{2 t}^{r s}(k)-\chi_{t}^{r s}\right)\right)}, \quad \forall t \in T^{r s}, r s \in \mathrm{RS}, k \in K
$$

where $q e_{2}^{r s}(k)$ is the number of combined-mode travelers during interval $k$ between OD pair $r s . \theta_{T}^{r s, t}$ is the parameter that reflects the travel cost sensitivity on transfer point choices of combined-mode travelers. $\chi_{t}^{r s}$ represents the relative attractiveness of transfer point $t$.

The EMC for combined-mode travelers during interval $k$ between OD pair $r s$ is given as follows:

$$
\mu_{2}^{r s}(k)=-\frac{1}{\theta_{T}^{r s, t}} \ln \left(\sum_{t \in T_{r s}} \exp \left(-\theta_{T}^{r s, t} \cdot\left(\mu_{2 t}^{r s}(k)-\chi_{t}^{r s}\right)\right)\right)=\mu_{2 t}^{r s}(k)-\chi_{t}^{r s}+\frac{1}{\theta_{T}^{r s, t}} \ln \left(\frac{q e_{2 t}^{r s}(k)}{q e_{2}^{r s}(k)}\right), \quad \forall k \in K, r s \in \mathrm{RS} .
$$

Finally, the departure time choice behaviors for combined-mode travelers can be expressed as follows:

$$
q e_{2}^{r s}(k)=q e_{2}^{r s} \frac{\exp \left(-\theta_{D}^{r s, t} \cdot \mu_{2}^{r s}(k)\right)}{\sum_{k \in K} \exp \left(-\theta_{D}^{r s, t} \cdot \mu_{2}^{r s}(k)\right)}, \quad \forall r s \in \mathrm{RS}, k \in K
$$

where $q e_{2}^{r s}$ is the number of combined-mode travelers between OD pair $r s$. The parameter $\theta_{D}^{r s, t}$ reflects the travel cost sensitivity on departure time choices. In order to ensure the validity of formulations (47)-(51), $\theta_{R}^{r s, t} \geq \theta_{T}^{r s, t} \geq \theta_{D}^{r s, t}$ must hold.

The EMC for combined-mode travelers between OD pair $r s$ is given as follows:

$$
\mu_{2}^{r s}=-\frac{1}{\theta_{D}^{r s, t}} \ln \left(\sum_{k \in K} \exp \left(-\theta_{D}^{r s, t} \cdot \mu_{2}^{r s}(k)\right)\right)=\mu_{2}^{r s}(k)+\frac{1}{\theta_{D}^{r s, t}} \ln \left(\frac{q e_{2}^{r s}(k) \cdot \delta}{q e_{2}^{r s}}\right), \quad \forall r s \in \mathrm{RS} .
$$

Substituting equations (48) and (50) into equation (52), we can obtain

$$
\mu_{2}^{r s}=C_{p t}^{r s}(k, \cdot)-\chi_{t}^{r s}+\frac{1}{\theta_{R}^{r s, t}} \ln \left(\frac{f_{p t}^{r s}(k)}{q_{2 t}^{r s}(k)}\right)+\frac{1}{\theta_{T}^{r s, t}} \ln \left(\frac{q e_{2 t}^{r s}(k)}{q e_{2}^{r s}(k)}\right)+\frac{1}{\theta_{D}^{r s, t}} \ln \left(\frac{q e_{2}^{r s}(k) \cdot \delta}{q e_{2}^{r s}}\right) .
$$

Substituting equations (47) and (49) into equation (53), we can obtain

$$
\begin{aligned}
& \mu_{2}^{r s}=\frac{\theta_{R}^{r s, t}}{\theta_{D}^{r s, t}} C_{p t}^{r s}(k, \cdot)+\frac{\theta_{R}^{r s, t}-\theta_{T}^{r s, t}}{\theta_{R}^{r s, t} \cdot \theta_{D}^{r s, t}} \ln \left(\sum_{p t \in P_{2 t}^{r s}} \exp \left(-\theta_{R}^{r s, t} \cdot C_{p t}^{r s}(k, \cdot)\right)\right) \\
& +\frac{\theta_{T}^{r s, t}-\theta_{D}^{r s, t}}{\theta_{T}^{r s, t} \cdot \theta_{D}^{r s, t}} \ln \left(\sum_{t \in T_{r s}} \exp \left(-\theta_{T}^{r s, t}\left(\frac{-1}{\theta_{R}^{r s, t}} \cdot \ln \left(\sum_{p t \in P_{2 t}^{r s}} \exp \left(-\theta_{R}^{r s, t} \cdot C_{p t}^{r s}(k, \cdot)\right)\right)-\chi_{t}^{r s}\right)\right)-\frac{\theta_{T}^{r s, t}}{\theta_{D}^{r s, t}} \chi_{t}^{r s}+\frac{1}{\theta_{D}^{r s, t}} \ln \left(\frac{f_{p t}^{r s}(k) \cdot \delta}{q e_{2}^{r s}}\right)\right.
\end{aligned}
$$


We follow the analysis of the travel choice behaviors for pure-mode travelers. For each generalized unit route travel cost of combined-mode travelers $C_{p t}^{r s}(k, \cdot)$, we define a so- called augmented travel cost that is equal to the right-hand side of equation (54).

$$
\begin{aligned}
\widehat{C}_{p t}^{r s}(k, \cdot)= & \frac{\theta_{R}^{r s, t}}{\theta_{D}^{r s, t}} C_{p t}^{r s}(k, \cdot)+\frac{\theta_{R}^{r s, t}-\theta_{T}^{r s, t}}{\theta_{R}^{r s, t} \cdot \theta_{D}^{r s, t}} \ln \left(\sum_{p t \in P_{2 t}^{r s}} \exp \left(-\theta_{R}^{r s, t} \cdot C_{p t}^{r s}(k, \cdot)\right)\right) \\
& +\frac{\theta_{T}^{r s, t}-\theta_{D}^{r s, t}}{\theta_{T}^{r s, t} \cdot \theta_{D}^{r s, t}} \ln \left(\sum_{t \in T_{r s}} \exp \left(-\theta_{T}^{r s, t}\left(\frac{-1}{\theta_{R}^{r s, t}} \cdot \ln \left(\sum_{p t \in P_{2 t}^{r s}} \exp \left(-\theta_{R}^{r s, t} \cdot C_{p t}^{r s}(k, \cdot)\right)\right)-\chi_{t}^{r s}\right)\right)-\frac{\theta_{T}^{r s, t}}{\theta_{D}^{r s, t}} \chi_{t}^{r s}+\frac{1}{\theta_{D}^{r s, t}} \ln \left(\frac{f_{p t}^{r s}(k) \cdot \delta}{q e_{2}^{r s}}\right) \cdot\right.
\end{aligned}
$$

Meanwhile, we can find the equalization of augmented travel cost $\widehat{C}_{p t}^{r s}(k, \cdot)$ for all actually used route $p$ via transfer point $t$, chosen transfer point, and selected departure time $k$ implies a nested-logit stochastic dynamic route, transfer

$$
\widehat{C}_{p t}^{r s}(k, \cdot)\left\{\begin{array}{l}
=\hat{C}_{t, \text { min }}^{r s} \text {, if } f_{p t}^{r s}(k)>0 \\
>\hat{C}_{t, \text { min }}^{r s} \text {, if } f_{p t}^{r s}(k)=0
\end{array} \quad \forall p t \in P_{2 t}^{r s}, t \in T^{r s}, k \in K, r s \in \mathrm{RS},\right.
$$

point, and departure time equilibrium solution as the one given by equations (47)-(51).

Therefore, the nested-logit stochastic dynamic route, transfer point, and departure time equilibrium condition for combined-mode travelers can be given as follows:

where $\widehat{\Omega}_{t}$ is a closed convex.

$$
\widehat{\Omega}_{t}=\left\{f_{t} \mid \sum_{\mathrm{p}} \sum_{\mathrm{k}} \sum_{t} f_{p t}^{r s *}(k)=\frac{q e_{2}^{r s}}{\delta}, f_{p t}^{r s}(k) \geq 0, \quad \forall r s\right\} .
$$

expanded travel cost for combined-mode travelers on any unused routes. The combined-mode travelers cannot alter departure time, transfer point, and route choices to diminish the expanded travel cost.

The above SUE-SRD equilibrium condition of combined-mode travelers can be expressed by a finite-dimensional variational inequality formulation.

Find a vector $\hat{f}_{t}^{*} \in \Omega_{t}$ if and only if it satisfies

$$
\sum_{r s} \sum_{p} \sum_{k} \sum_{t} \widehat{C}_{p t}^{r s}\left(k, f, f_{t}^{*}\right)\left(f_{p t}^{r s}(k)-f_{p t}^{r s *}(k)\right) \geq 0 \quad \forall \widehat{f} \in \widehat{\Omega},
$$

where $\widehat{C}_{t, \min }^{r s}=\min \left\{\widehat{C}_{p t}^{r s}(k, \cdot), p t \in P_{2 t}^{r s}, t \epsilon^{r s}, k \in K\right\}$. Under the equilibrium condition, the augmented travel cost experienced by all combined-mode travelers between OD pair $r s$ is equal and minimum and less than (or equal to) the (57) and only if it satisfies the VI problem:

\section{.}

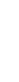

$$
\sum_{r s} \sum_{p} \sum_{k} \widehat{C}_{p}^{r s}\left(k, f^{*}, f_{t}^{*}\right)\left(f_{p}^{r s}(k)-f_{p}^{r s *}(k)\right)+\sum_{r s} \sum_{p} \sum_{k} \sum_{t} \widehat{C}_{p t}^{r s}\left(k, f^{*}, f_{t}^{*}\right)\left(f_{p t}^{r s}(k)-f_{p t}^{r s *}(k)\right) \geq 0, \quad \forall f^{*} \in \widehat{\Omega}, \forall f_{t}^{*} \in \widetilde{\Omega}_{t},
$$

Here, we discuss the property of the solution to VI (59). Huang [11] proved that the route travel cost functions are continuous in route inflow rates for the simultaneous route and departure time user equilibrium problem with single user class. The dynamic link model in this paper is similar to the point queue model proposed by Huang and Williams (2001). Thus, we consider that the continuity of the generalized unit route travel cost functions for pure-mode and combined-mode travelers holds. Since $\left.\widehat{C}_{p t}^{r s} k, \cdot\right)$ and $\widehat{C}_{p t}^{r s}(k, \cdot)$ are continuous and $\Omega$ is the compact convex set, VI (59) admits at least a solution. In general, the monotonicity of the generalized unit route travel cost functions cannot hold; therefore, $\left.\widehat{C}_{p t}^{r s} k, \cdot\right)$ and $\widehat{C}_{p t}^{r s}(k, \cdot)$ are not monotonic too. Further, there exist multiple solutions in VI (59). 


\section{Algorithms}

In this section, we propose a heuristic algorithm to solve VI (59). The proposed algorithm is heuristic in that convergence cannot be proved. Details of the heuristic algorithm are described as follows.

Step.1 (Initialization): Choose initial route flow patterns $f_{p}^{r s}(k)_{(i)}, f_{p t}^{r s}(k)_{(i)}$. Set the iteration index $i=1$.

Step.2 (Dynamic network loading): Perform the dynamic network loading as described in (1)-(28) according to the route flow pattern. Obtain the corresponding route travel cost $C_{p}^{r s}(k)_{(i)}, C_{p t}^{r s}(k)_{(i)}$, respectively.

Step.3 (Auxiliary route flow): Assign the demand of pure-mode travelers $q e_{1(i)}^{r s}$ to the route by using equations (38) and (40), and obtain the auxiliary route flow rates of pure-mode travelers $f_{p}^{r s}(k)_{(i)}^{*}$. Assign the demand of pure-mode travelers $q e_{2(i)}^{r s}$ to the route via transfer link by using equations (47), (49), and (51), and obtain the auxiliary route flow rates of combined-mode travelers $f_{p t}^{r s}(k)_{(i)}^{*}$.

Step.4 (Method of successive average):

$$
\begin{aligned}
& f_{p t}^{r s}(k)_{(i+1)}=f_{p t}^{r s}(k)_{(i)}+\frac{1}{i}\left(f_{p t}^{r s}(k)_{(i)}^{*}-f_{p t}^{r s}(k)_{(i)}\right), \quad \forall p t \in P_{2 t}^{r s}, t \in T^{r s}, k \in K, r s \in \mathrm{RS}, \\
& f_{p}^{r s}(k)_{(i+1)}=f_{p}^{r s}(k)_{(i)}+\frac{1}{i}\left(f_{p}^{r s}(k)_{(i)}^{*}-f_{p}^{r s}(k)_{(i)}\right), \quad \forall p \in P_{1}^{r s}, k \in K, r s \in \mathrm{RS} .
\end{aligned}
$$

Step.5 (Convergence): If

$$
\sum_{r s} \sum_{k} \sum_{p} f_{p}^{r s}(k)\left[\frac{\left.\widehat{C}_{p}^{r s}(k, \cdot)-\widehat{C}_{\min }^{r s}(\cdot)\right]+\sum_{r s} \sum_{k} \sum_{p} \sum_{t} f_{p t}^{r s}(k)\left[\widehat{C}_{p t}^{r s}(k, \cdot)-\widehat{C}_{t, \text { min }}^{r s}(\cdot)\right]}{\sum_{r s} \sum_{k} \sum_{p} f_{p}^{r s}(k) \widehat{C}_{\min }^{r s}(\cdot)+\sum_{r s} \sum_{k} \sum_{p} \sum_{t} f_{p t}^{r s}(k) \widehat{C}_{t, \min }^{r s}(\cdot) \leq \xi, \quad \text { stop }} ;\right.
$$

otherwise, set $i=i+1$; then go to Step 2 .

\section{Simulation Examples}

The example network in Figure 2 consists of 9 nodes, 12 links, 2 bus lines, and 1 OD pair $(1,9)$.

The route sets of pure-mode and combined-mode travelers between OD pair $(1,9)$ are shown in Figure 3. The data associated with roads and transfer links of the network are given in Table 1 . These bus stops are not marked with a superscript " to avoid the confusion. Two bus lines are No.1 line $=\{5,6,9\}$ and No.2 line $=\{5,8,9\}$. Hence, there are three transfer points $\{5,6,8\}$. The dispatching origins of two bus lines are node 5. Let the dispatching frequency of two bus lines be 12 (veh/h), and the capacity of the bus is 50 persons. The nonadditive bus fares (RMB/person) are as follows: for bus lines No.1 and No.2, $\rho_{5-6-9}=\rho_{5-8-9}=1, \rho_{6-9}=\rho_{8-9}=$ 0.5. The demands for pure-mode and combined-mode travelers are 10000 and 4000, respectively.

The example network with symmetric structure shows that some routes and links will have the same traffic condition, such as route and link inflow rate [11]. For pure-mode travelers, the traffic conditions on routes 1 and 6 and routes $2,3,4$, and 5 are identical. For combined-mode travelers, the traffic conditions on routes $2,3,4$, and 5 via transfer point 5 , on route 1 via transfer point 6 , and route 6 via transfer point 8 , on routes 2 and 4 via transfer point 6 , and on routes 3 and 5 via transfer point 8 are identical. Thus, we will discuss the numerical results on routes 1 and 2 for pure-mode travelers, route 2 via transfer point 5 , route 1 via transfer point 6 , and route 2 via transfer point 6 for combined-mode travelers.

The passenger car equivalents parameters of car and bus are $P c u=1$ and $P c u_{\mathrm{b}}=2$. Other input data are $\lambda_{2}=0.5, \beta=8$, $\alpha_{1}=\alpha_{2}=\gamma_{t}=10, \gamma=22, \Delta_{\mathrm{m}}=0.25 \mathrm{~h}$, and $k_{m}{ }^{*}=9.0 \mathrm{~h}$. The study period is composed of four hours, from $6: 00$ am to 10 : $00 \mathrm{am}$, which is discretized into 400 intervals with $\delta=0.6 \mathrm{~min}$.

The data associated with travel choices is as follows:

$$
\begin{aligned}
& \theta_{R}^{19}=1, \theta_{R}^{19, t}=1, \theta_{T}^{19, t}=0.08, \theta_{D}^{19}=0.5, \theta_{D}^{19, t}=0.5, \\
& \lambda_{1}^{19}=1, \lambda_{1}^{19}=5, \lambda_{1 N}^{19}=14 .
\end{aligned}
$$

The value of the convergence indicator is generally decreasing as the iteration proceeds, although oscillations occur. While the iteration number amounts to 50, the value of the convergence indicator has a very small change in Figure 3. Figure 4 gives the route inflow rates and the augmented travel costs on routes 1 and 2 for pure-mode travelers. We can find an approximate dynamic equilibrium pattern for all route inflow rates of pure-mode travelers between OD pairs $(1,9)$. Figure 5 shows the route inflow rates and the augmented travel costs on route 2 via transfer point 5 , route 1 via transfer point 6 , and route 2 via transfer point 6 for combined-mode travelers. From Figure 6, we can see that the 


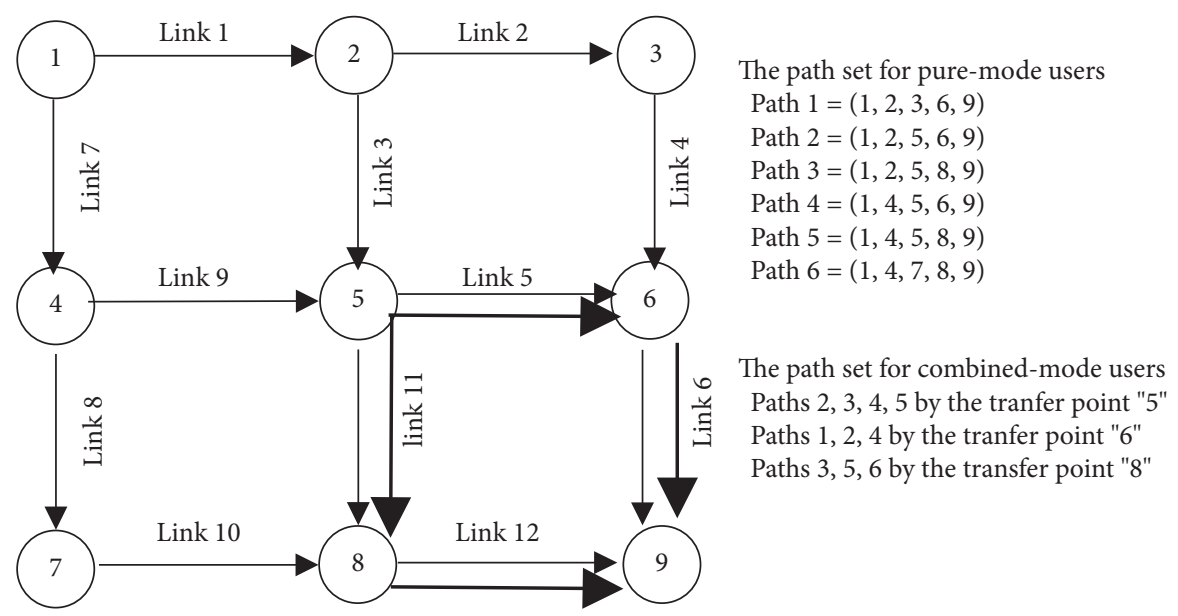

FIgURE 2: The example network.

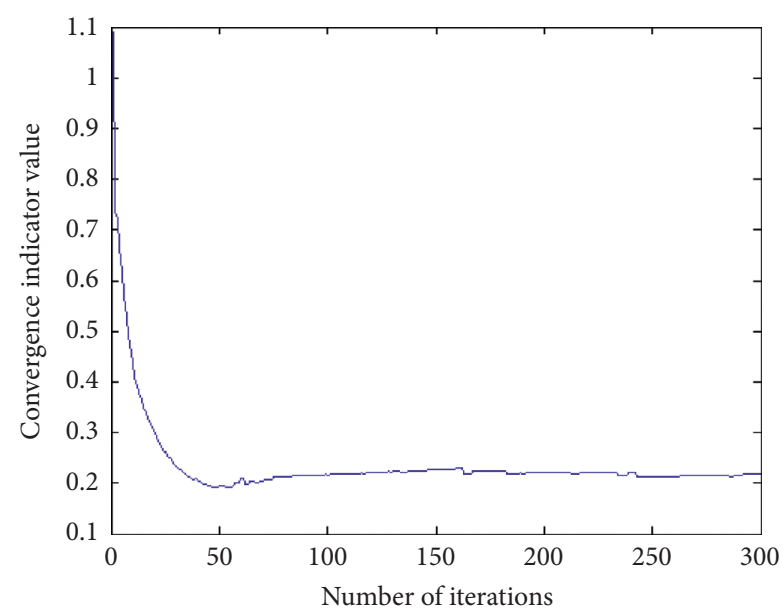

FIgURE 3: Convergence indicator.

TABLe 1: The link data.

\begin{tabular}{lccccc}
\hline Road network & & & & \multicolumn{2}{c}{ Transfer link } \\
$L_{a}$ & $t_{a}^{c}$ & $t_{a}^{b}$ & $S_{a}$ & $L_{t t_{1}}$ & $S_{t t_{1}}$ \\
\hline 1 & 0.2 & & 4000 & $55^{\prime}$ & 0.01 \\
2 & 0.15 & & 2000 & 66 & 0.01 \\
3 & 0.2 & & 2500 & & 0.01 \\
4 & 0.15 & & 2000 & \\
5 & 0.1 & 0.15 & 2000 & & \\
6 & 0.2 & 0.3 & 3000 & & \\
7 & 0.2 & & 4000 & & \\
8 & 0.15 & & 2000 & & \\
9 & 0.2 & & 2500 & & \\
10 & 0.15 & & 2000 & & \\
11 & 0.1 & 0.15 & 2000 & & \\
12 & 0.2 & 0.3 & 3000 & & \\
\hline
\end{tabular}

equalization of the augmented travel costs for the chosen route via transfer point, transfer point, and departure time can be obtained. In other words, the stochastic dynamic user equilibrium for a route via transfer point, transfer point, and departure time choices of combined-mode travelers can be obtained.
Figure 6 shows the queue length of a car on some links at all intervals. The queuing peaks mainly occur on links 1 and 7. There are few queues on links 3(9), 5(11), and 6(12). Links $2,4,6$, and 10 have a null queue. There are no passenger queues on transfer links $55^{\prime}, 66^{\prime}$, and $88^{\prime}$. The queues of 


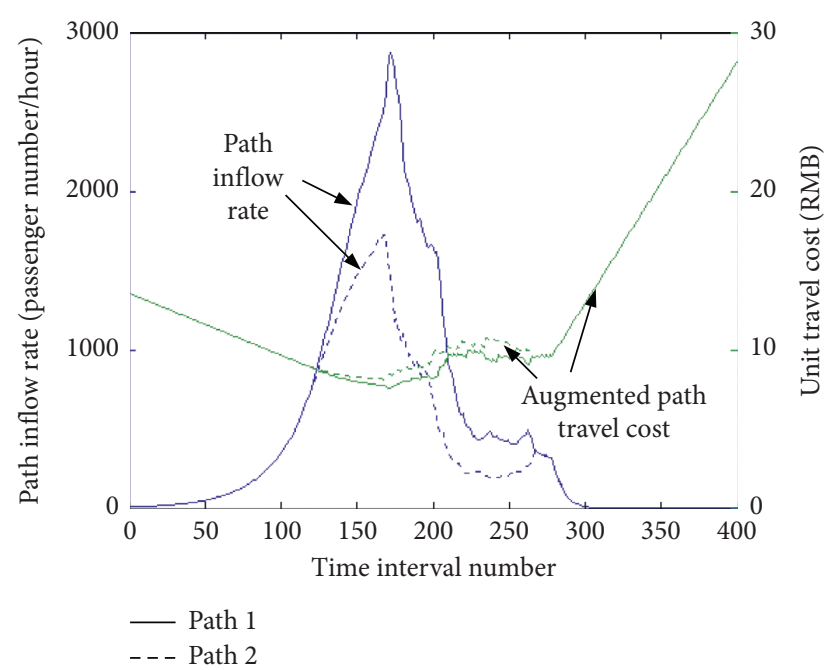

FIgURE 4: The route inflow rates and the augmented travel costs for pure-mode travelers.

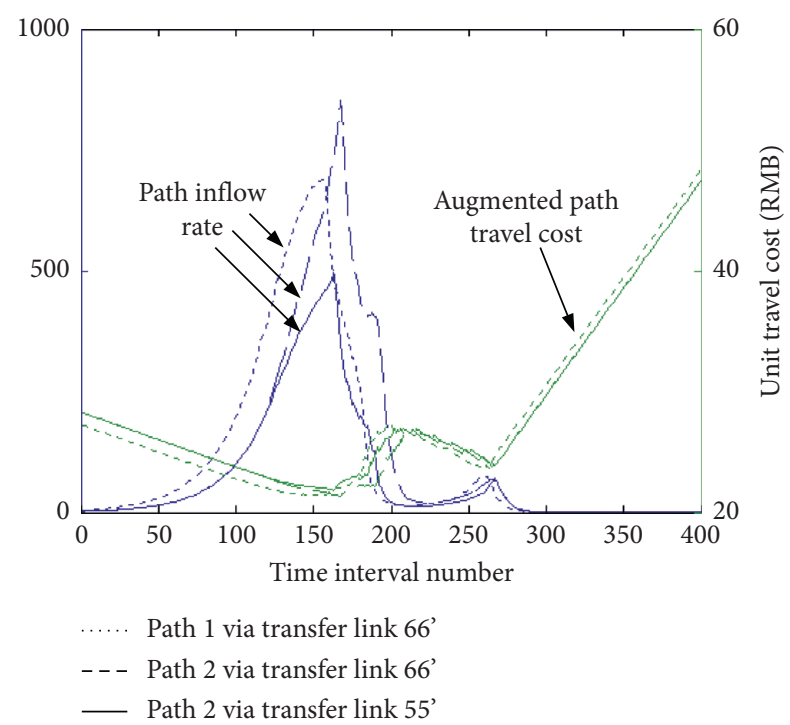

Figure 5: The route inflow rates and the augmented travel costs for combined-mode travelers.

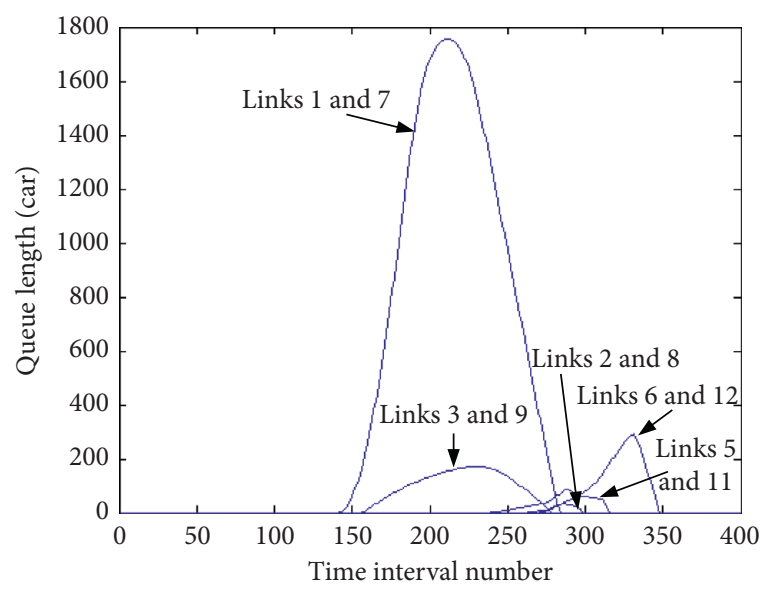

Figure 6: Link queue. buses on links $5,6,11$, and 12 are few due to the small bus dispatching frequency at the origin of the bus.

\section{Conclusions}

In this paper, we propose an integrated variational inequality formulation to model dynamic network equilibrium conditions with combined mode. We classify travelers into two classes: one is pure-mode travelers, and another is combinedmode travelers. We use the nested-logit-type functions to capture the complex traveler choice behaviors for two classes. Finally, using a simple example demonstrates the validity of the heuristic algorithms and model.

Future studies may focus on the extensions of the proposed model, including individual transfer choices and the model parameters calibrated. The approach in this paper may be used to assess the impacts of various traffic management policies such as parking lot planning and park fare [14-17].

\section{Data Availability}

The data used to support the findings of this study have not been made available because the data are proprietary or confidential in nature and may only be provided with restrictions.

\section{Conflicts of Interest}

The authors declare that they do not have any commercial or associative interest that represents conflicts of interest in connection with the work submitted.

\section{Acknowledgments}

Financial support provided by the Fundamental Research Funds for the Central Universities, CHD (300102328402 and 300102320201), is acknowledged. This research was supported by Shaanxi Provincial Natural Science Foundation of China under Grant no. 2016JM5052 and Xi'an Science and Technology Bureau Project Funding (2019218514GXRC021CG022GXYD21.3.

\section{References}

[1] M. C. J. Bliemer, Analytical dynamic traffic assignment with interaction user-classes: theoretical advances and applications using a variational inequality approach, Ph.D. thesis, Delft University of Technology, Delft, Netherlands, 2001.

[2] M. C. J. Bliemer and P. H. L. Bovy, "Quasi-variational inequality formulation of the multiclass dynamic traffic assignment problem," Transportation Research Part B: Methodological, vol. 37, no. 6, pp. 501-519, 2003.

[3] E. Fernandez, J. De Cea, M. Florian, and E. Cabrera, "Network equilibrium models with combined modes," Transportation Science, vol. 28, pp. 182-192, 1994.

[4] R. García and A. Marín, "Parking capacity and pricing in Park 'n ride trips: a continuous equilibrium network design problem," Annals of Operations Research, vol. 116, no. 1/4, pp. 153-178, 2002.

[5] H. J. Huang, Z.-C. Li. 2003. A variational inequality formulation for modeling a two-mode elastic demand 
transportation system with Park'n ride trips. Submitted for Transportation Science.

[6] S. Kim, A. Chen, and X. Xu, "Modeling mode and route similarities in network equilibrium problem with go-green modes," Networks and Spatial Economics, vol. 16, no. 1, pp. 33-60, 2016.

[7] S. Ryu and A. Chen, "Alternate weibit-based model for assessing green transport systems with combined mode and route travel choices," Transportation Research Part B: Methodological, vol. 103, pp. 291-310, 2017.

[8] Z. Liu, X. Chen, and Q. Meng, "Remote park-and-ride network equilibrium model and its applications," Transportation Research Part B: Methodological, vol. 117, pp. 37-62, 2018.

[9] S. G. Li and Y. M. Su, "Multimode stochastic dynamic simultaneous route/departure time equilibrium problem with queues," Journal of the Eastern Asia Society for Transportation Studies, vol. 6, pp. 2092-2107, 2005.

[10] S. G. Li and Y. F. Ju, "Evaluation of bus exclusive lane," IEEE Transaction on Intelligent Transportation System, vol. 10, no. 2, pp. 236-245, 2009a.

[11] H.-J. Huang and W. H. K. Lam, "Modeling and solving the dynamic user equilibrium route and departure time choice problem in network with queues," Transportation Research Part B: Methodological, vol. 36, no. 3, pp. 253-273, 2002.

[12] J. Li and O. Fujiwara, "A reactive dynamic user equilibrium model in network with queues," Transportation Research Part B: Methodological, vol. 34, no. 8, pp. 605-624, 2000.

[13] W. H. K. Kawakami and H. J. Huang, "A multi-class dynamic user equilibrium model for queuing networks with advanced traveler information systems," Journal of Mathematical Modelling and Algorithms, vol. 2, pp. 349-377, 2003.

[14] S. Han, "Dynamic traffic modelling and dynamic stochastic user equilibrium assignment for general road networks," Transportation Research Part B: Methodological, vol. 37, no. 3, pp. 225-249, 2003.

[15] H. K. Lo, C. W. Yip, and K. H. Wan, "Modeling transfer and non-linear fare structure in multi-modal network," Transportation Research Part B: Methodological, vol. 37, no. 2, pp. 149-170, 2003.

[16] M. Kuwahara and T. Akamatsu, "Decomposition of the reactive dynamic assignments with queues for a many-to-many origin-destination pattern," Transportation Research Part B: Methodological, vol. 31, no. 1, pp. 1-10, 1997.

[17] W. H. K. Lam, Z. Li, S. C. Wong, and D. Zhu, "Modeling an elastic-demand bimodal transport network with park-andride trips," Tsinghua Science and Technology, vol. 12, no. 2, pp. 158-16610, 2007. 